\title{
Impacto de la Geometría en el Efecto Fin de Generadores Lineales
}

\author{
Alejandro Tapia-Hernández ${ }^{(1)}$, Mario Ponce-Silva ${ }^{(1)}$, Nancy Mondragón-Escamilla ${ }^{(2)}$, Leobardo \\ Hernández-González (2) \\ (1) Centro Nacional de Investigación y Desarrollo Tecnológico, Departamento de Ingeniería Electrónica, \\ Interior Internado Palmira SN, Col. Palmira, C.P. 62490 Cuernavaca, Morelos - México. \\ (2) Instituto Politécnico Nacional ESIME Culhuacán. Av. IPN S/N, México D.F. - México \\ (e-mail: alejandro.tapia.hernandez@gmail.com; ponce@cenidet.edu.mx; naytsu10@hotmail.com; \\ Ihernandezg@ipn.mx).
}

Recibido Nov. 5, 2015; Aceptado Ene. 12, 2016; Versión final Mar. 1, 2016, Publicado Ago. 2016

\begin{abstract}
Resumen
En este artículo se presentan resultados experimentales para un generador lineal con diferentes condiciones de efecto fin con el fin de obtener una comparación cualitativa para ajustar los parámetros geométricos del generador (longitud de los devanados y longitud del estator). El uso de generadores lineales en los sistemas de aprovechamiento de energía es algo común hoy en día. Uno de los problemas que presentan estos generadores es la distorsión en la forma de onda del voltaje inducido debido al "efecto fin". El efecto fin se presenta debido al cambio de velocidad que presenta el rotor en los bordes del generador y repercute de forma directa en la forma de onda del voltaje inducido y en la transferencia de energía hacia la carga. Sin embargo, el efecto fin no es un factor que se incluya en la metodología de diseño de los generadores lineales. La propuesta de este trabajo permite obtener una forma de onda senoidal de voltaje inducido en lugar de una forma de onda pulsante de voltaje inducido como se ha reportado en la literatura.
\end{abstract}

Palabras clave: generador lineal; efecto fin; inducción magnética; ondas de voltaje

\section{Impact of Geometry on Linear Generators End-Effect}

\begin{abstract}
In this paper experimental results for a linear generator with different end-effect constraints to obtain a qualitative comparison for adjusting the linear generator geometry (windings length and stator length), are presented. The use of linear generators in energy harvesting systems is very common nowadays. One of the problems of this kind of electric generators is an induced voltage distorted due to end-effects. The end-effect is produced by the rotor speed change at the borders of the electric generator and impacts directly on the induced voltage waveform and in the energy flux to the load. However, is not included usually on the methodology design of linear generators. The proposal presented in this work allows obtaining a sinusoidal induced voltage waveform instead of a pulsing induce voltage waveform as has been reported in literature
\end{abstract}

Keywords: linear generator; end effect; magnetic induction; voltage waves 


\section{INTRODUCCIÓN}

La mayoría de los esquemas para el aprovechamiento de energía que se reportan en la literatura, típicamente se basan en el aprovechamiento de la energía cinética del medio ambiente y para esto emplean en su mayoría generadores de imanes permanentes dadas sus ventajas en peso y costo frente a generadores con rotor devanado. Existen dos geometrías básicas de generadores de imanes permanentes, los generadores lineales y los generadores rotacionales. Los generadores lineales se aplican principalmente a esquemas que aprovechan la energía del mar, vibraciones mecánicas o el movimiento del cuerpo humano por nombrar los más representativos (Yuan et al. 2011, Ekström et al. 2015 and Gao et al. 2016). Este tipo de generadores ofrecen varias ventajas respecto a los generadores rotacionales como lo son: un bajo peso, una alta eficiencia en la generación de energía eléctrica, disminución de pérdidas por fricción respecto a esquemas rotantes, un diseño compacto y una alta densidad de potencia en función de la geometría empleada. A diferencia de las metodologías de diseño que se aplican en los generadores comerciales, en las aplicaciones para el aprovechamiento de energía los parámetros más importantes y que se buscan optimizar son la frecuencia y la distorsión de la forma de onda del voltaje inducido.

Un generador ideal es capaz de entregar una frecuencia elevada con una forma de onda de voltaje inducido aproximada a una forma de onda sinusoidal. Aunque hoy en día se ha popularizado el uso de los generadores lineales para aplicaciones de aprovechamiento de energía, los parámetros empleados en su diseño consideran únicamente el desempeño mecánico del generador y no el impacto de estos en su desempeño eléctrico, específicamente en la calidad de la forma de onda de voltaje inducido. Algunas de las metodologías de diseño reportadas para generadores lineales son: Li et al. (2011) y Gao et al. (2016). Los trabajos anteriores consideran en la metodología de diseño del generador lineal dos parámetros principales; la frecuencia y la magnitud del voltaje inducido. La frecuencia depende directamente de la velocidad de desplazamiento del rotor y del número de polos del estator, sin embargo, para obtener una mejor aproximación de la magnitud real del voltaje inducido Vermaak and Kamper (2012) consideran que el voltaje inducido depende de varios parámetros indirectos como lo son el número de devanados y polos del generador, el número de vueltas de los devanados, y de manera adicional del espaciamiento entre los imanes permanentes y los devanados ya que esto modifica la densidad de flujo magnético que actúa sobre los devanados. Ya que el principio de operación del generador lineal es la inducción magnética, las variaciones de velocidad tienen un impacto importante en el comportamiento del voltaje inducido. Debido a que la magnitud del voltaje inducido es función de la velocidad del campo magnético, cualquier cambio de velocidad tiene un efecto directo en la magnitud y aún más importante en la forma de onda del voltaje inducido. El cambio más brusco de velocidad del campo magnético se presenta en los bordes del generador lineal debido a la naturaleza de su operación, a esté fenómeno de cambio de velocidad en los bordes del generador se le conoce como "efecto fin".

Debido al efecto fin, el cambio de velocidad provoca una distorsión en la forma de onda del voltaje inducido ocasionando que la forma de onda del voltaje inducido tenga un mayor tiempo con voltaje nulo en el devanado, por lo tanto solo se aprecian picos de energía aprovechable que no permiten una transferencia constante de energía lo que reduce la eficiencia del generador. En la literatura se ha explorado el efecto fin de los generadores lineales, por ejemplo, Yang et al. (2011) considera el efecto fin aplicado a motores lineales de inducción únicamente para conocer el comportamiento del campo magnético en el espaciamiento entre los cilindros pero no se analiza su impacto en la calidad de la forma de onda de voltaje inducido. Creppe and Ulson (2008) analizan el efecto fin en motores lineales para determinar su comportamiento fuerza contra velocidad sin verificar su impacto en la forma de onda del voltaje inducido. A pesar de la importancia y el impacto que tiene el efecto fin en el comportamiento de los generadores lineales, este solo se considera en el diseño de motores para modelar su comportamiento buscando la optimización de la longitud del motor, no obstante, no se han reportado trabajos que exploren el impacto del efecto fin en la calidad de la forma de onda de voltaje inducido, aportación principal de este trabajo. La aportación principal de este trabajo es realizar un análisis cualitativo de la distorsión en la forma de onda del voltaje inducido de generadores lineales ocasionada por el efecto fin, considerando el impacto de la geometría de diseño del generador para evitar o minimizar la distorsión de la forma de onda de voltaje inducido, manteniendo una forma de onda aproximada a una senoidal.

\section{INDUCCIÓN MAGNÉTICA Y REPERCUSIONES EN LA FORMA DE ONDA}

La Fig. 1 muestra el diagrama básico de un generador lineal. Este se encuentra formado de dos partes principales: un cilindro fijo que contiene un arreglo de devanados en serie (estator) y un cilindro móvil que contiene un arreglo de imanes permanentes (rotor). El espaciamiento entre ambos cilindros (rotor y estator) tiene un efecto directo en la magnitud del voltaje inducido tal como menciona Yang et al. (2011). El fenómeno de inducción magnética se produce como consecuencia de las variaciones de campo magnético que actúan sobre los devanados que forman el estator. El movimiento del rotor para el ejemplo de la Fig. 1 es de izquierda a derecha y viceversa usando los resortes en los bordes del generador como un 
amortiguador para el movimiento del rotor. Como consecuencia del movimiento lineal del rotor en el generador y de la distribución de los devanados, este tipo de generadores emplean típicamente imanes axiales en el rotor, es decir que su flujo es perpendicular a la superficie plana del imán (ver Fig. 1). Usando imanes axiales se tiene un mejor aprovechamiento de las líneas de flujo magnético y una fácil implementación en sistemas multipolos debido a la atracción natural de los imanes.

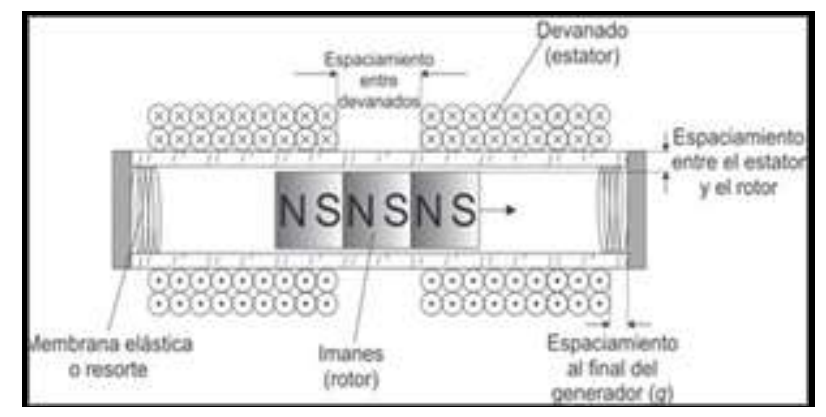

Fig. 1: Estructura del generador lineal

El comportamiento del generador lineal se basa en las leyes generales del electromagnetismo (ley de Faraday y ley de Ampere) tal como se muestra en los trabajos de Creppe and Ulson (2008), Shiri and Shoulaie (2012), Alonge et al. (2015) y Gao et al. (2016), Haciendo uso de la expresión de Lorentz (1) es posible simplificar el cálculo del voltaje inducido en el generador, reduciendo su cálculo a una sola expresión. La expresión de Lorentz describe el comportamiento de campos magnéticos cambiantes en el tiempo y la inducción magnética que provocan en los devanados del generador lineal. De acuerdo a la ecuación (1), que es la forma simplificada de la expresión de Lorentz, los parámetros que determinan el nivel del voltaje inducido son la velocidad lineal aplicada al rotor formado por los imanes permanentes $(v)$, la densidad del campo magnético que está dada por las características magnéticas del imán empleado $(\beta)$, el número de vueltas que atraviesa el campo magnético del rotor $(N)$ y la longitud de cada espira del devanado que cruza el campo magnético la cual corresponde a la longitud de una vuelta del estator $(L)$.

$\mathcal{E}=v N \beta L$

De los parámetros que se emplean en (1) para el cálculo del voltaje inducido, $L$ es constante, $\beta$ varía con el espaciamiento entre el rotor y el estator pero es fijo para una geometría especifica de generador lineal ya que en ningún momento se modifica la distancia entre el rotor y el estator, $N$ es fijo ya que no se modifica el número de vueltas del estator, no obstante la cantidad de espiras que atraviesan el cambio magnético si es función del desplazamiento del rotor y dependen de la geometría del generador, y finalmente $v$ varía con el tiempo ya que existe una aceleración y desaceleración del rotor al llegar a ambos extremos del generador lineal (efecto fin). Esta desaceleración del rotor se debe a la operación natural del generador lineal, y se suaviza al colocar en ambos extremos del generador lineal un resorte, una membrana flexible o un imán con polaridad opuesta a la del rotor para reducir el impacto del rotor al llegar al final del generador. Para calcular el valor de $\beta$, en Fei et al. (2010) se muestra un modelo analítico que describe el comportamiento de la densidad de flujo magnético de los imanes en función del espaciamiento entre ellos y el devanado del estator. En la Fig. 2 se muestra el comportamiento de la densidad de flujo magnético de los imanes del rotor para una geometría circular de $10 \mathrm{~mm}$ de diámetro hechos de una aleación de neodimio N35. Como se puede observar en la Fig. 2, al incrementar el espaciamiento entre los imanes del rotor y los devanados, se reduce de manera considerable la densidad de flujo magnético de los imanes debido a que se incrementa la cantidad de flujo disperso.

Para describir el comportamiento de la velocidad del rotor en el generador lineal no existe un comportamiento generalizado, ya que depende de varios factores: la posición del generador en operación (vertical u horizontal), la membrana o amortiguador empleado en los bordes del generador y el mecanismo que se emplee para mover el rotor del generador lineal. En la Fig. 3 se muestran los cambios en la velocidad del rotor durante su operación natural dentro del generador lineal considerando un desplazamiento inicial de izquierda a derecha (a) y el cambio a un desplazamiento de derecha a izquierda (b). En ambos casos se alcanza un máximo de velocidad que se mantiene por un tiempo determinado, y posteriormente empieza una desaceleración ocasionada por la membrana o amortiguador en el borde del generador lineal. De la Fig. 3 se puede observar que la velocidad del rotor varía en función de la distancia, y tiene un cambio más significativo en los bordes del generador debido al efecto fin y a la cantidad de espiras que se aprovechan del devanado para generar el voltaje inducido. De acuerdo a la ecuación (1) se puede observar que el cambio de velocidad y del número de espiras es proporcional al cambio en la magnitud del voltaje inducido, por lo que a partir de estos dos parámetros es posible determinar el grado de deformación que presentará la forma de onda del voltaje inducido como consecuencia del efecto fin. 


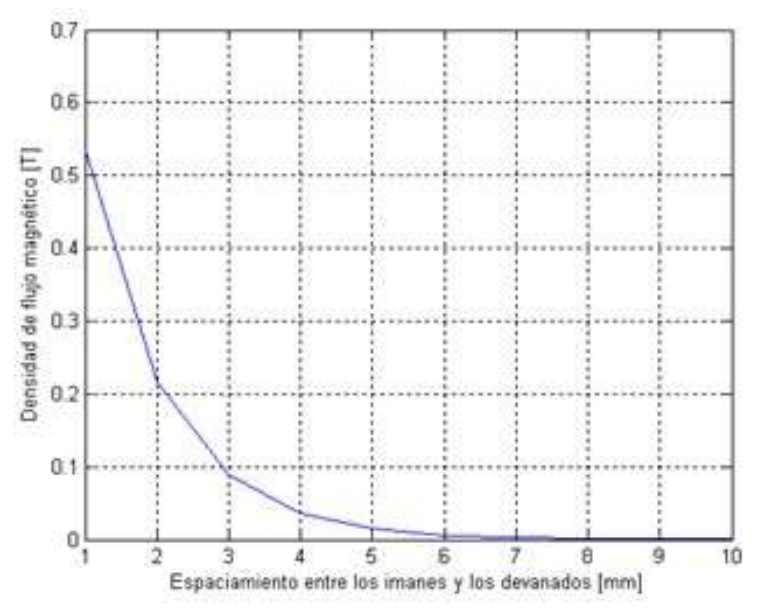

Fig. 2: Comportamiento de la densidad de flujo magnético de los imanes en función del espaciamiento entre los imanes y los devanados

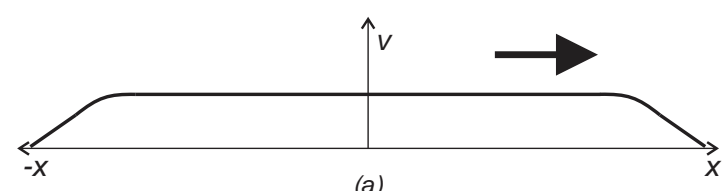

(a)

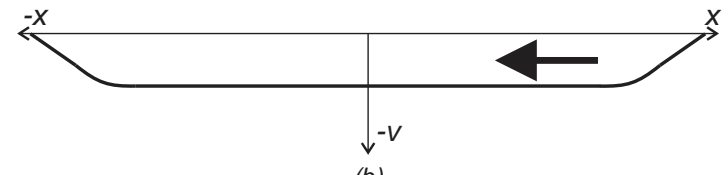

(b)

Fig. 3: Comportamiento de la velocidad del rotor para un desplazamiento de izquierda a derecha (a) y de derecha a izquierda $(b$

En la Fig. 4 se muestra como se produce la inducción magnética en un devanado del generador lineal considerando dos configuraciones típicas: (a) se tienen un devanado que no cubre por completo al rotor en los bordes del generador lineal y (b) se tiene un devanado que cubre por completo el rotor en los bordes del generador lineal.

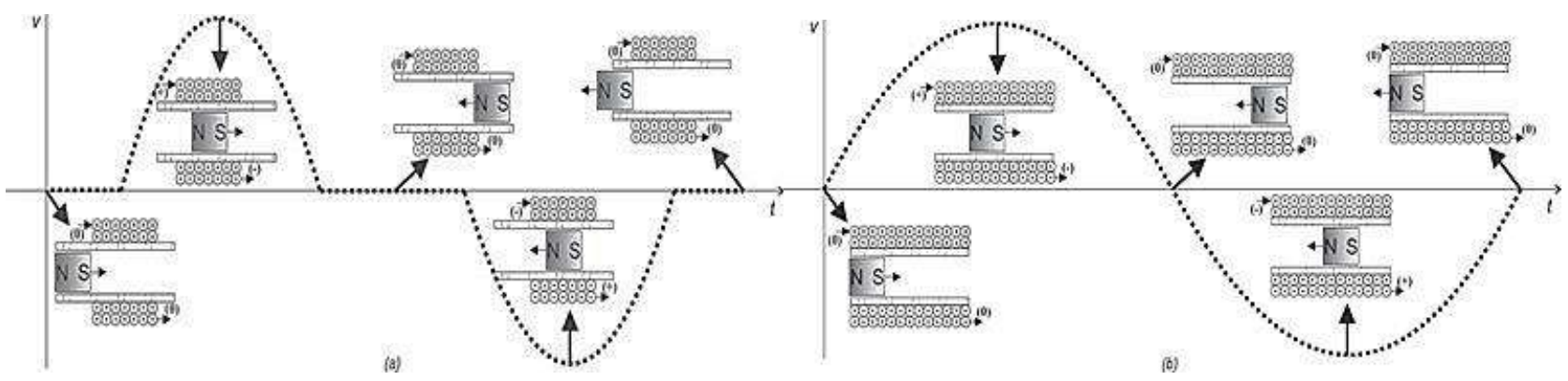

Fig. 4: Voltaje inducido para diferentes posiciones del rotor: (a) un devanado que no cubre los bordes del generador lineal y (b) un devanado que cubre los bordes del generador lineal.

De la Fig. 4a se puede observar que cuando el imán (rotor) cruza el devanado, se genera un voltaje inducido cuya polaridad depende de la dirección de movimiento con respecto a la polaridad del imán, por lo que se obtiene el voltaje máximo o mínimo cuando el imán se encuentra a la mitad del devanado ya que se tiene la máxima condición de velocidad y máxima cantidad de espiras del devanado. Mientras el rotor sigue su desplazamiento (hacia la izquierda o derecha) el voltaje inducido en el devanado disminuye gradualmente ya que la cantidad de espiras excitadas por el flujo magnético se reducen. Cuando el rotor ya ha atravesado todo el devanado, el voltaje inducido en las terminales del generador es nulo ya que no existe inducción magnética en el devanado, esto genera una forma de onda pulsante de voltaje inducido tal como muestra la Fig. 4a. En la Fig. 4b se puede observar un comportamiento similar al de la Fig. 4a para la inducción de los voltajes máximos y mínimos de la forma de onda de voltaje inducido al igual que en el decremento del voltaje. La diferencia en el comportamiento de las dos configuraciones se tiene en los bordes del generador lineal, a diferencia de la Fig. $4 \mathrm{a}$, en la Fig. $4 \mathrm{~b}$ no se tienen voltajes nulos de inducción en los bordes ya que todo el tiempo se obtienen espiras excitadas por el flujo magnético del rotor. Esto ocasiona que únicamente se tenga un voltaje nulo cuando el rotor alcanza una velocidad cero. De acuerdo a esto podemos concluir que entre más tiempo pasa el rotor fuera del devanado, se incrementa el tiempo en que el voltaje inducido es nulo, es decir que si se tiene un devanado que abarque mas área del generador lineal en especial en los bordes del mismo, se reduce el tiempo en que el voltaje inducido es nulo ya que en todo momento existe inducción magnética en el devanado. En la siguiente sección se detallará el diseño del prototipo de generador lineal empleado para corroborar el fenómeno descrito en las secciones anteriores.

\section{RESULTADOS}

Para validar la hipótesis descrita en las secciones anteriores, se construyó un prototipo de generador lineal para ser usado con un motor Stirling con velocidad nominal de $2000 \mathrm{rpm}$ y con un concentrador parabólico, el generador lineal se muestra en la Fig. 5 y sus dimensiones en la Tabla 1 para obtener un voltaje inducido pico de $200 \mathrm{mV}$ a la velocidad nominal. 
Tabla 1: Dimensiones del generador lineal construido.

\begin{tabular}{|l|c|}
\hline Parámetro & Valor \\
\hline Longitud del generador & $124 \mathrm{~mm}$ \\
\hline Número de devanados & 2 \\
\hline Longitud de cada devanado & $47 \mathrm{~mm}$ \\
\hline Número de vueltas por devanado & 100 \\
\hline Distancia entre los devanados & $10 \mathrm{~mm}$ \\
\hline Longitud del rotor & $19.5 \mathrm{~mm}$ \\
\hline Diámetro de los imanes & $10 \mathrm{~mm}$ \\
\hline Diámetro del generador lineal & $12.52 \mathrm{~mm}$ \\
\hline
\end{tabular}

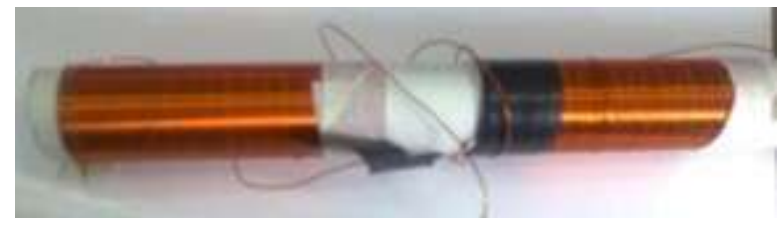

Fig. 5: Prototipo del generador lineal.

La distancia entre los devanados de acuerdo a Zheng et al. (2007) es igual a la mitad de la longitud del rotor $(10 \mathrm{~mm})$. Las pruebas experimentales realizadas al generador lineal se enfocaron únicamente en la comparación cualitativa de la forma de onda del voltaje inducido, esto con el fin de ver el impacto que tienen diferentes condiciones de espaciamiento en los bordes del generador tal como se describió en la sección anterior (efecto fin). Para realizar la prueba se empleó una membrana elástica instalada en ambos bordes del generador que se acondiciono para permitir variar la longitud efectiva del generador lineal y con ello variar el espaciamiento entre el rotor y los bordes del generador. Los casos analizados fueron tres: en el primero los devanados no cubren el desplazamiento del rotor en los bordes del generador lineal por lo que no tiene espiras para inducir voltaje, en el segundo los devanados en el borde del generador lineal cubren parcialmente al rotor en su desplazamiento final y en el tercero los devanados cubren totalmente el desplazamiento del rotor en los bordes del generador lineal. Usando el mismo espaciamiento usado entre los devanados $(10 \mathrm{~mm})$ como parámetro de diseño de las tres configuraciones propuestas. La Fig. 6 muestra los voltajes inducidos obtenidos para las tres condiciones de espaciamiento en el generador lineal: con un espaciamiento al final del devanado de $10 \mathrm{~mm}$ (Fig. 6a), sin espaciamiento (Fig. 6b) y con una reducción de $-10 \mathrm{~mm}$ (Fig. 6c), es decir que aun en el borde el rotor se encuentra cubierto por el devanado del estator.

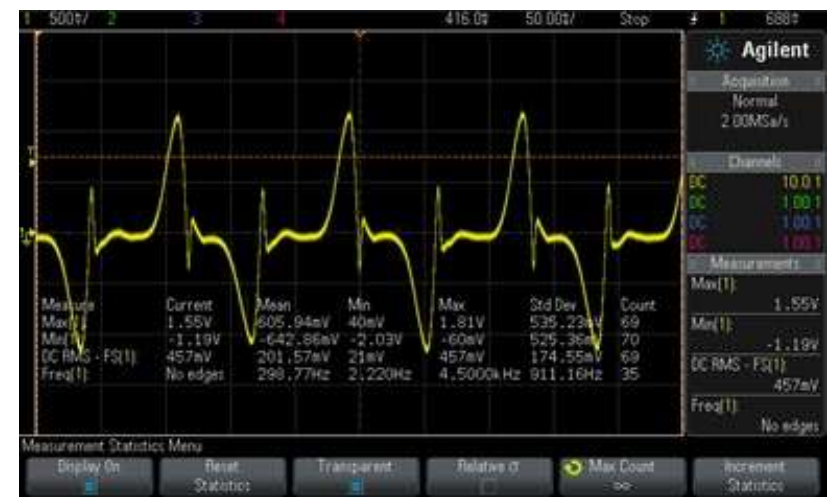

(a)

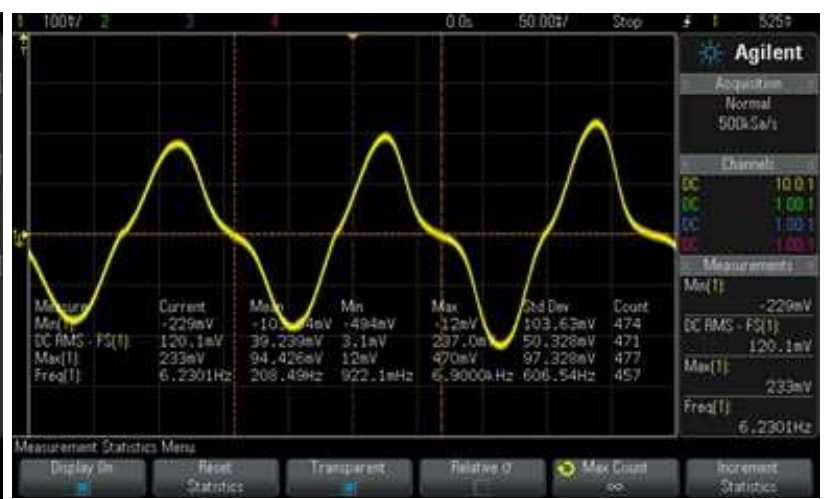

(b)

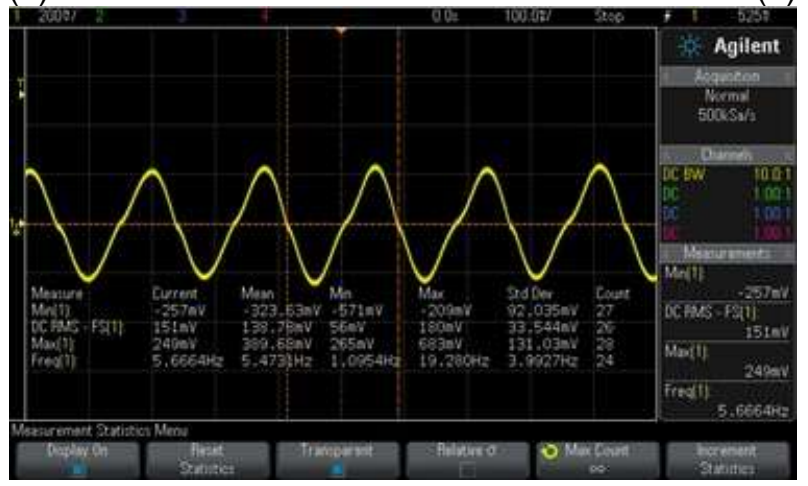

(c)

Fig. 6: Resultados experimentales para espaciamientos de: (a) $10 \mathrm{~mm}$ (1V/div, $50 \mathrm{~ms} / \mathrm{div})$, (b) sin espacio final (100 mV, $50 \mathrm{~ms} / \mathrm{div})$ y (c) $-10 \mathrm{~mm}(200 \mathrm{mV}, 100 \mathrm{~ms} / \mathrm{div})$. 
De los resultados obtenidos se puede observar que en la Fig. 6a el voltaje inducido se presenta durante un corto periodo de tiempo, esto dificulta su aprovechamiento ya que se tienen grandes cantidades de energía en un corto tiempo. En la Fig. 6b se puede observar que al disminuir el espaciamiento en los bordes del generador se logra reducir el lapso de tiempo en que el voltaje inducido es nulo lo que ayuda a tener una mejor transferencia de energía ya que el voltaje se encuentra distribuido en el periodo de la forma de onda. Finalmente cuando el espaciamiento es negativo, es decir que el rotor se encuentra cubierto totalmente por el devanado, se eliminan los lapsos en que el voltaje inducido es nulo o distorsionado, por lo que la forma de onda se asemeja más a una forma de onda sinusoidal (Fig. 6c). En la operación de los generadores lineales lo más recomendable es que la forma de onda de voltaje inducido se asemeje a una forma de onda sinusoidal, de lo contrario al concentrar toda la energía del generador en pequeños lapsos de tiempo se tienen mayores esfuerzos eléctricos en los dispositivos del convertidor de potencia asociado a la operación del generador. En la medida que se tenga una distribución uniforme de la energía en el tiempo, se garantizará una operación eficaz del convertidor.

\section{CONCLUSIONES}

Con los resultados obtenidos se hizo una comparación cualitativa de tres configuraciones de generadores lineales que buscan resaltar el efecto que tiene la geometría del generador en la calidad de la forma de onda del voltaje inducido. De estos resultados se observa que si se requiere una transferencia constante de energía para minimizar el uso de dispositivos de almacenamiento en el convertidor electrónico asociado al generador lineal, será necesario reducir la región de desplazamiento de los imanes aunque esto signifique reducir el voltaje inducido, o bien, se puede optar por incrementar el área cubierta por el devanado para mantener la magnitud del voltaje inducido deseado con una misma región de desplazamiento del rotor. Integrando estas consideraciones dentro del diseño de la geometría del generador, será posible mantener una transferencia constante de energía del generador lineal con una forma de onda de voltaje inducido senoidal en el estator.

\section{AGRADECIMIENTOS}

Los autores agradecen al Consejo Nacional de Ciencia y Tecnología (CONACYT) de México, al Instituto Politécnico Nacional (IPN) y al Tecnológico Nacional de México (TecNM) por su apoyo económico.

\section{REFERENCIAS}

Alonge, F., y otros 3 autores, Input-output feedback linearizing control of linear induction motor taking into consideration the end-effects. Part II: Simulation and experimental results. Control Engineering Practice, 36 , 142-150. (2015)

Creppe, R.C. y Ulson, C., Influence of Design Parameters on Linear Induction Motor End Effect. IEEE Transactions on Energy Conversion, 23(2), 358-362 (2008)

Ekström, R., Ekergård, B. y Leijon, M., Electrical damping of linear generators for wave energy convertersA review. Renewable and Sustainable Energy Reviews, 42, 116-128. (2015)

Fei, W., Luk, P.C.K. y Jinupun, K., Design and analysis of high-speed coreless axial flux permanent magnet generator with circular magnets and coils. IET Electric Power Applications, 4(9), p.739 (2010)

Gao, Y., y otros 5 autores, A fully floating system for a wave energy converter with direct-driven linear generator. Energy, 95, 99-109. (2016)

Li, W., Chau, K.T. y Jiang, J.Z., Application of Linear Magnetic Gears for Pseudo-Direct-Drive Oceanic Wave Energy Harvesting. IEEE Transactions on Magnetics, 47(10), 2624-2627 (2011)

Shiri, A. y Shoulaie, A., End effect braking force reduction in high-speed single-sided linear induction machine. Energy Conversion and Management, 61, 43-50 (2012)

Vermaak, R. y Kamper, M.J., Design Aspects of a Novel Topology Air-Cored Permanent Magnet Linear Generator for Direct Drive Wave Energy Converters. IEEE Transactions on Industrial Electronics, 59(5), 2104-2115 (2012)

Yang, T., Zhou, L. y Li, L., Influence of Design Parameters on End Effect in Long Primary Double-Sided Linear Induction Motor. IEEE Transactions on Plasma Science, 39(1), 192-197 (2011)

Yuan, B., y otros 5 autores, A Current-Fed Multiresonant Converter with Low Circulating Energy and ZeroCurrent Switching for High Step-Up Power Conversion. IEEE Transactions on Power Electronics, 26(6), 1613-1619. (2011)

Zheng, P., y otros 4 autores, Research on a Tubular Longitudinal Flux PM Linear Generator Used for FreePiston Energy Converter. IEEE Transactions on Magnetics, 43(1), 2006-2008 (2007) 